\title{
A rare presentation of nephrotic syndrome
}

\author{
Debabrata Mukherjee $^{1} \cdot$ Sourabh Sharma $^{1} \cdot$ Debashish Mahapatra $^{1}$. \\ Ranjith K. Nair ${ }^{1}$ - Bhaskar Datt ${ }^{1}$ - Dhawal Arora ${ }^{1}$
}

Received: 6 April 2017 / Accepted: 27 June 2017/Published online: 30 June 2017

(C) Japanese Society of Nephrology 2017

\begin{abstract}
Nephrotic syndrome usually presents with oedema due to fluid accumulation in subcutaneous space. Renal subcapsular fluid accumulation is a rare presentation. We present a rare case of membranoproliferative glomerulonephritis which presented with bilateral subcapsular fluid collection and was successfully managed by percutaneous drainage and treating the underline pathology.
\end{abstract}

Keywords Membranoproliferative glomerulonephritis . Subcapsular fluid collection · Floating kidney

\section{Introduction}

Nephrotic syndrome usually presents with oedema due to fluid accumulation in subcutaneous space. Renal subcapsular fluid accumulation is a rare presentation. We present a rare case of membranoproliferative glomerulonephritis which presented with bilateral subcapsular fluid collection and was managed successfully by treating the underlying pathology.

\section{Case report}

A 15-year-old male presented with left-sided flank pain for 2 weeks. He also had history of facial puffiness since last 3 months. On detailed evaluation, he was found to have

Sourabh Sharma

drsourabh05@gmail.com

1 Army Hospital Research and Referral, Dhaula Kuan, Delhi 110010, India hypertension (blood pressure 160/96 mmHg, right arm supine) and fullness in left flank. He also had hypoalbuminemia (serum albumin $1800 \mathrm{mg} / \mathrm{dl}$ ), nephrotic range proteinuria (6500 mg/day) and microscopic hematuria. His renal functions and lipid profile were normal. Coagulation profile and liver function tests were normal. Serum C3 and C4 levels within normal range while antinuclear antibody (ANA), anti ds DNA antibody, perinuclear anti-neutrophil cytoplasmic antibodies (p-ANCA), cytoplasmic anti-neutrophil cytoplasmic antibodies (c-ANCA) were negative. Viral markers (hepatitis B surface antigen and anti-hepatitis C antibody) and anti HIV-I and II were negative. Ultrasonography abdomen showed bilateral perinephric collection. Contrast-enhanced computed tomography (CECT) scan of abdomen revealed large left renal subcapsular collection with deformed renal contour and mild perinephric collection on right side (Fig. 1a, b). Ultrasonography guided pigtail aspiration of left-sided collection was done and 1.51 of transudative fluid was removed. Kidney biopsy was performed thereafter which revealed eleven glomeruli, all showing variable degree of mesangial and endothelial proliferation. Three glomeruli showed lobular accentuation of glomerular tuft with the presence of mesangial and endothelial hypercellularity. Splitting of glomerular basement membrane was also seen. The tubules showed few hyaline and granular casts. There was no significant interstitial fibrosis or tubular atrophy. Blood vessels were unremarkable. Immunofluorescence study showed IgA 1+, mesangial and capillary wall, granular; IgG 2+, segmental mesangial, granular; IgM trace, segmental mesangial, granular; C3 1+ mesangial and capillary wall, granular; C1q negative. Biopsy findings were suggestive of membranoproliferative glomerulonephritis (Fig. 2a, b). He was started on steroids and achieved 

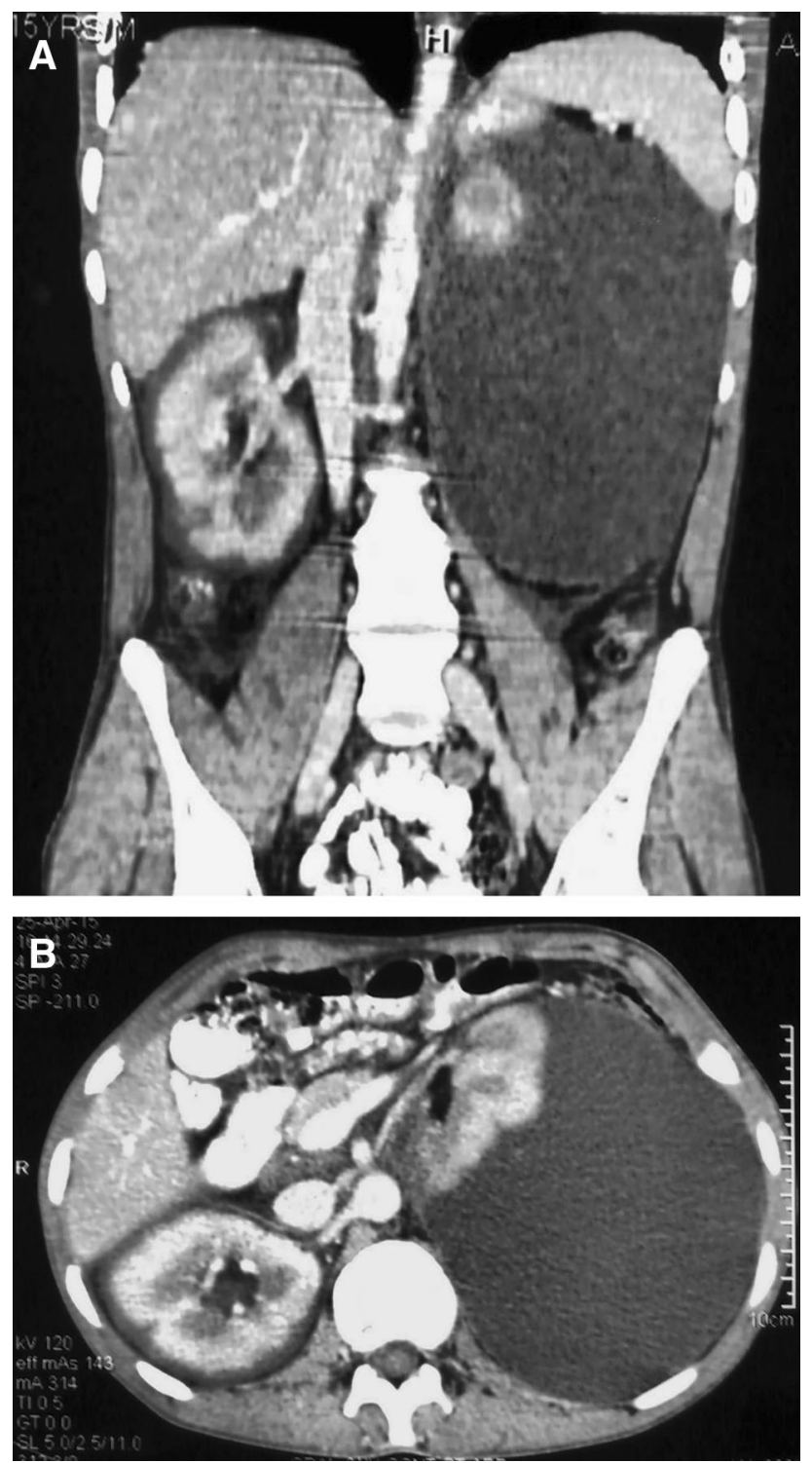

Fig. 1 Contrast-enhanced CT image (axial (a) and coronal (b) sections) showing large left-sided subcapsular fluid collection with deformed renal contour and mild right-sided perinephric collection

remission after 4 weeks. There was no recurrence of fluid collection on repeat ultrasonography 2 months later.

\section{Discussion}

Renal subcapsular fluid collection is commonly encountered by radiologists due to accumulation of blood, pus or serous fluid in subcapsular space. It is usually postoperative or traumatic urine leak and pancreatitis. It is rarely a presenting feature of nephrotic syndrome which is classically characterized by fluid accumulation in subcutaneous space or in third spaces of body like pleura, peritoneum and perineum.
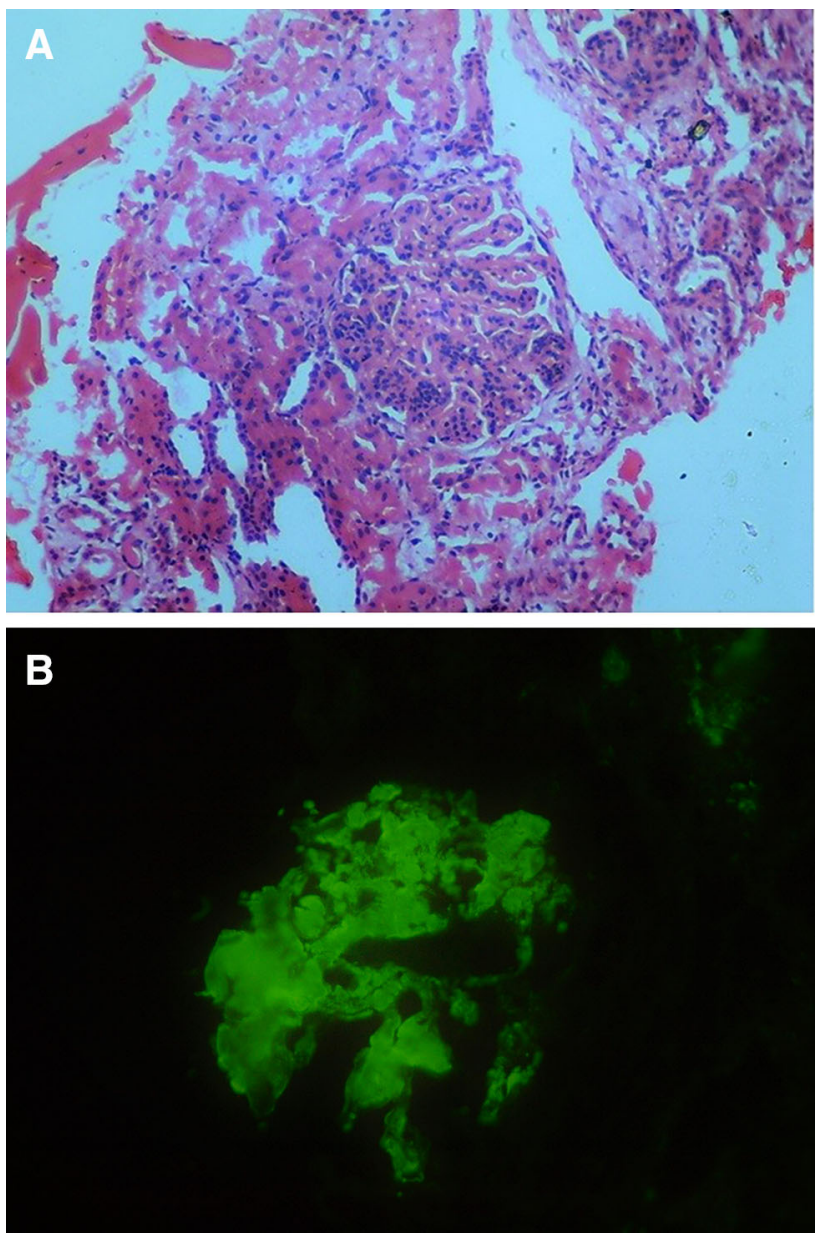

Fig. 2 Light microscopy (a) of biopsy specimen (H\&E), showing membranoproliferative glomerulopathy. IF study (b) shows segmental deposition of $\operatorname{IgG}$ along mesangial which is granular in appearance

The kidneys are surrounded by a tough capsule that is separate from the underlying cortex. There is a potential subcapsular space between the capsule and cortex. Subcapsular fluid collections are visible as crescentic-shaped collections on ultrasonography $[1,2]$. According to the volume of fluid, subcapsular fluid collections are graded into three ultrasonographic patterns. Grade 1 is a thin layer of fluid around the kidney, grade 2 is a moderate effusion with indentations of the underlying renal parenchyma, and grade 3 is a large fluid collection surrounding the kidney [3].

On detailed review of literature, we could find only three case reports, out of which two were focal segmental glomerulosclerosis (FSGS) $[1,4]$ and one was membranous glomerulonephritis (MGN) [5]. Yalcin et al. [4] and Wani et al. [1] reported cases of FSGS which presented with subcapsular fluid collection and was successfully treated with percutaneous aspiration followed by immunosuppressant (steroids and cyclophosphamide). Orofino et al. [5] reported a case of MGN with renal vein thrombosis 
which presented with subcapsular fluid accumulation and was treated with povidone iodine instillation into renal capsule. This is first case of membranoproliferative glomerulonephritis presenting with bilateral subcapsular fluid collection. We reiterate through this case that perinephric subcapsular fluid collection can be a rare complication of nephrotic syndrome. It can be successfully managed by percutaneous drainage and treating the underline pathology.

\section{Compliance with ethical standards}

Conflict of interest The authors have declared that no conflict of interest exists.

Research involving human participants and/or animals This article does not contain any studies with human participants or animals performed by any of the authors.

Informed consent Informed consent was obtained from all individual participants included in the study.

\section{References}

1. Wani MA, Mir F, et al. Subcapsular fluid collection: an unusual manifestation of nephrotic syndrome. Am J Kidney Dis. 2010;56(1):181-4.

2. McCune TR, Stone WJ, et al. Page kidney: case report and review of literature. Am J Kidney Dis. 1991;18(5):593-9.

3. Haddad MC, Medawar WA, et al. Perirenal fluid in renal parenchymal medical disease ('floating kidney'): clinical significance and sonographic grading. Clin Radiol. 2001;56(12):979-83.

4. Yalcin AU, Akcar N, et al. An unusual presentation for nephrotic syndrome: bilateral perirenal subcapsular fluid collection. Nephron. 2002;92:244-5.

5. Orofino L, Herrero JA, et al. Perirenal subcapsular fluid collection in a patient with membranous nephropathy and renal vein thrombosis. J Urol. 1986;136:1287-9. 